\title{
Management of COVID-19 in people with epilepsy: drug considerations
}

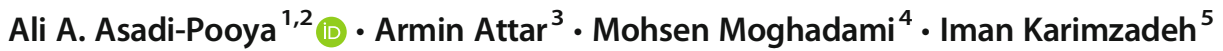

Received: 8 June 2020 / Accepted: 21 June 2020 / Published online: 27 June 2020

(C) Fondazione Società Italiana di Neurologia 2020

\begin{abstract}
People with epilepsy (PWE) are neither more likely to be infected by the coronavirus nor are they more likely to have severe COVID-19 manifestations because they suffer from epilepsy. However, management of COVID-19 in PWE may be more complicated than that in other individuals. Drug-drug interactions could pose significant challenges and cardiac, hepatic, or renal problems, which may happen in patients with severe COVID-19, may require adjustment to antiepileptic drugs (AEDs). In this review, we first summarize the potential drug-drug interactions between AEDs and drugs currently used in the management of COVID-19. We then summarize other challenging issues that may happen in PWE, who have COVID-19 and are receiving treatment.
\end{abstract}

Keywords Antiepileptic drug $\cdot$ COVID $\cdot$ Epilepsy $\cdot$ Interaction $\cdot$ Seizure

\section{Introduction}

Recently, the world has been experiencing a catastrophic phenomenon, a pandemic of coronavirus disease (COVID-19) that is caused by SARS-CoV2 [1]. This virus primarily targets the human respiratory system, but coronaviruses also have

Ali A. Asadi-Pooya

aliasadipooya@yahoo.com

Armin Attar

attar_armin@yahoo.com

Mohsen Moghadami

mohsen168@gmail.com

Iman Karimzadeh

karimzadehiman@yahoo.com

1 Epilepsy Research Center, Shiraz University of Medical Sciences, Shiraz, Iran

2 Jefferson Comprehensive Epilepsy Center, Department of Neurology, Thomas Jefferson University, Philadelphia, PA, USA

3 Department of Cardiovascular Medicine, Shiraz University of Medical Sciences, Shiraz, Iran

4 Health Policy Research Center, Institute of Health, Shiraz University of Medical Sciences, Shiraz, Iran

5 Department of Clinical Pharmacy, Shiraz University of Medical Sciences, Shiraz, Iran neuroinvasive capabilities and can spread from the respiratory tract to the central nervous system (CNS). COVID-19 has been associated with neurological manifestations such as seizures and change in mental status [2].

Available information on the pandemic suggests that people with epilepsy (PWE) are neither more likely to be infected by the coronavirus nor are they more likely to have severe COVID-19 manifestations because they suffer from epilepsy [3]. However, management of COVID-19 in PWE may be more complicated than that in other individuals.

While, so far no therapy has been approved for the treatment of COVID-19, there are more than a handful of therapies under investigation and being used. Therefore, drug-drug interactions between anti-COVID therapies and antiepileptic drugs (AEDs) could pose significant challenges. In addition, cardiac, hepatic, or renal problems, which may happen in patients with severe COVID-19, may require adjustment to AEDs. In this review, we first summarize the potential drug-drug interactions between AEDs and some of the drugs currently used in the management of COVID-19. We then summarize other challenging issues that may happen in PWE, who have COVID-19 and are receiving treatment.

\section{Drug interactions}

The mainstay of treatment of epilepsy is therapy with AEDs; nowadays, there are more than 30 AEDs available to treat 
epilepsy. Drug-drug interactions may occur whenever two or more drugs are administered simultaneously. Most clinically important, AED interactions with other drugs result from induction or inhibition of drug-metabolizing hepatic enzymes. Unfortunately, many AEDs are substrates, inducers, or inhibitors of hepatic enzymes; hence, drug-drug interactions are common in PWE [4-6]. Table 1 shows AED potentials for interactions with other drugs, in general. Table 2 shows the effects of anti-seizure medications and anti-COVID medications on hepatic enzymes and enzymes involved in their metabolism [6-16]. This table will help us understand the potential for drug interactions between AEDs and therapies suggested and used in COVID-19 (e.g., chloroquine, hydroxychloroquine, lopinavir/ritonavir, favipiravir, remdesivir, tocilizumab, nitazoxanide, sofosbuvir, and interferon beta). Since the treatment of COVID-19 is a changing landscape, the table of drug interactions could also be used as a roadmap when new therapies emerge.

While for many of the listed potential drug interactions, there is a lack of corresponding clinical evidence, it is important to consider these potential interactions and monitor the patient for possibility of inadequate efficacy or alternatively, toxicity of drugs. In other words, in any patient with epilepsy, who is being treated for COVID-19, therapeutic drug monitoring could provide valuable information to adjust the drug dosages. Also, close clinical monitoring of the patient may be warranted to ensure adequate efficacy and limit toxicity. Below is a summary of potential drug-drug interactions in these patients:

\section{Interactions resulting in decreased AED plasma levels}

Lopinavir/ritonavir decreases the plasma concentrations of lamotrigine (and possibly, phenytoin and valproate), probably by induction of glucuronidation enzyme system. A dose increment to $200 \%$ of the initial lamotrigine dose is needed to achieve concentrations similar to those with lamotrigine alone [17]. The therapeutic efficacy of many AEDs (e.g., carbamazepine, lacosamide, oxcarbazepine, lamotrigine, phenobarbital, phenytoin) may be decreased when used in combination with hydroxychloroquine or chloroquine [7,8]. The definite mechanism of this interaction has not been elucidated yet. The activity of many AEDs (e.g., carbamazepine, lacosamide, perampanel, phenobarbital, phenytoin) might be impaired if co-administered with tocilizumab, through hepatic enzyme induction (Table 2) [9].

\section{Interactions resulting in increased AED plasma levels}

Ritonavir is a potent inhibitor of CYP3A/ CYP2D6 and may potentially increase plasma levels of cannabidiol, carbamazepine, cenobamate, clonazepam, ethosuximide, lacosamide, perampanel, and zonisamide (Table 2) $[6,10,11]$.

\section{Interactions resulting in decreased plasma levels of other drugs}

Patients treated with enzyme-inducing AEDs (e.g., carbamazepine, cenobamate, eslicarbazepine acetate, oxcarbazepine, phenobarbital/pyrimidine, phenytoin) are at risk of important drug interactions with other drugs that are metabolized by these enzymes (e.g., hydroxychloroquine/chloroquine) (Table 2) [5, 6]. Increasing the dose of chloroquine/ hydroxychloroquine and also close monitoring of the patient's clinical response may be helpful in the circumstance of coadministration of these drugs. However, the needed amount of increase in chloroquine/hydroxychloroquine daily dose for compensating of this drug-interaction has not been defined. CYP3A inducers (e.g., carbamazepine, oxcarbazepine, eslicarbazepine, cenobamate, phenobarbital, phenytoin, topiramate) may also decrease lopinavir serum levels [12]. Co-administration of sofosbuvir with carbamazepine, phenytoin, phenobarbital, or oxcarbazepine is expected to decrease the serum concentration of sofosbuvir [13].

\section{Interactions resulting in increased plasma levels of other drugs}

Co-administration of hydroxychloroquine with moderate and strong CYP2C8 and CYP3A4 inhibitors (such as cannabidiol) may result in increased plasma concentrations of
Table 1 Anti-seizure medications potentials for interactions with other drugs

\begin{tabular}{ll}
\hline Drug interaction potential & Anti-seizure medications \\
\hline No significant drug interactions & Levetiracetam, brivaracetam*, pregabalin, vigabatrin, and gabapentin \\
$\begin{array}{c}\text { Mild to moderate potential for } \\
\text { drug interactions }\end{array}$ & $\begin{array}{c}\text { Eslicarbazepine acetate, oxcarbazepine, lacosamide, lamotrigine, clobazam, } \\
\text { perampanel, rufinamide, tiagabine, topiramate, and zonisamide } \\
\text { Phignificant drug interactions } \\
\text { Phobarbital, phenytoin, carbamazepine, primidone, cannabidiol, } \\
\text { cenobamate, and valproate }\end{array}$ \\
\hline
\end{tabular}

Reference: [6]

*Brivaracetam may have some drug interactions with tocilizumab 
Table 2 Effects of anti-seizure medications and anti-COVID medications on hepatic enzymes and enzymes involved in their metabolism

Effect

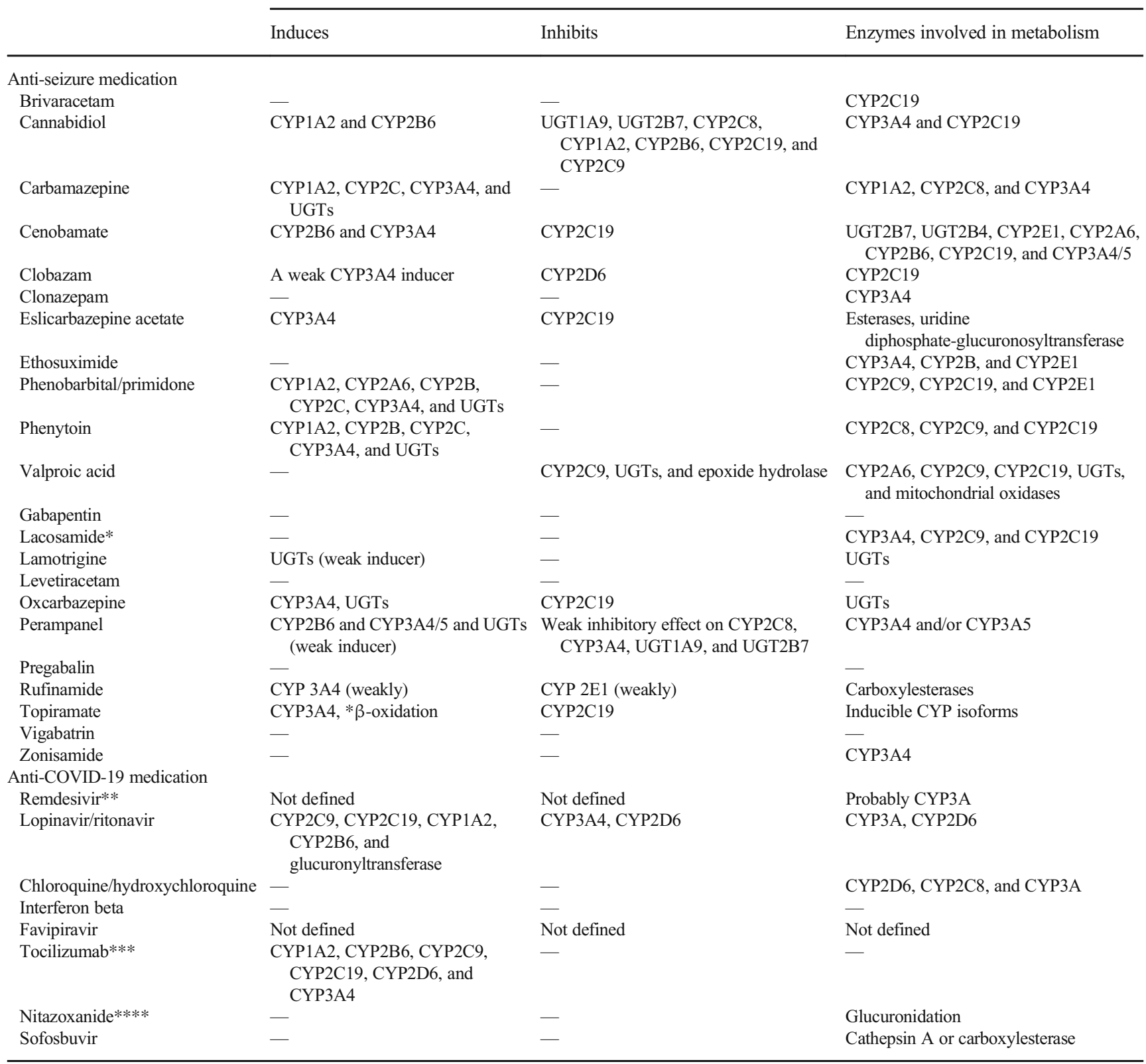

References: [6-16]

*Lacosamide should be used with caution in patients with known cardiac conduction problems (e.g., AV block and sick sinus syndrome without pacemaker), sodium channelopathies (e.g., Brugada syndrome), on concomitant medications that prolong PR interval, or with severe cardiac disease such as myocardial ischemia or heart failure. In such patients, obtaining an ECG before beginning lacosamide, and after lacosamide is titrated to steadystate, is recommended. Lacosamide administration may predispose the patient to atrial arrhythmias (atrial fibrillation or flutter), especially in those with diabetic neuropathy and/or cardiovascular disease [14]

**Hepatic enzyme inducers (e.g., carbamazepine, phenobarbital, phenytoin) may decrease remdesivir efficacy [15]

***Cytochrome P450s in the liver are downregulated by infection and inflammation stimuli including cytokines such as IL-6. Inhibition of IL-6 signaling in patients treated with tocilizumab may restore CYP450 activities to higher levels than those in the absence of tocilizumab leading to increased metabolism of drugs that are CYP450 substrates (including many AEDs) [9]

****Following oral administration in humans, nitazoxanide is rapidly hydrolyzed to an active metabolite, tizoxanide. Tizoxanide is highly bound to plasma protein (>99.9\%). Therefore, caution should be used when administering nitazoxanide concurrently with other highly plasma protein-bound drugs with narrow therapeutic indices (e.g., phenytoin) [16] 
hydroxychloroquine [8]. Perampanel is a weak CYP2C8 and CYP3A4 inhibitor (Table 2).

Note: Currently, there is neither experimental nor clinical evidence for any noticeable drug interactions between favipiravir, nitazoxanide, and interferon beta with AEDs.

\section{Adverse effects and other complications}

Many of the therapies currently in use for the treatment of COVID-19 have significant cardiovascular adverse effects. Similarly, many AEDs have significant cardiovascular adverse effects (Table 3) [7-16, 18-21]. Furthermore, COVID19 can cause systemic inflammation, leading to multi-organ dysfunction. Acute respiratory distress syndrome and respiratory failure, acute cardiac injury, and heart failure were the most common critical complications during exacerbation of COVID-19 [22]. All these potential drug adverse effects and medical complications of COVID-19 may complicate the management process of a critically ill patient.

When a patient with epilepsy becomes ill with COVID-19, there are two possible scenarios:

1. The patient has been well-controlled on a stable dose of $\operatorname{AED}(\mathrm{s})$, which happens in about two-thirds of the people with epilepsy, in general;

2. The patient has had drug-resistant seizures, which happens in about one-third of the patients with epilepsy.

In the first scenario, it is not practical to change the $\mathrm{AED}(\mathrm{s})$ suddenly even if there are significant drug-drug interactions and/or adverse effects related to AEDs. Therefore, the treating physician should be aware of any potential drug interactions, adverse effects, and complications related to AED(s) and/or anti-COVID treatments. Therapeutic drug monitoring could provide valuable information to adjust the drug regimen in these patients. Also, close monitoring of the patient (frequent history taking and physical examinations) may be warranted to ensure efficacy and limit toxicity of therapeutic interventions. Finally, actions to minimize cardiovascular risks (e.g., arrhythmia) in these circumstances may include [21, 23]:

- Electrocardiographic (ECG)/ QT interval monitoring.

- Correction of any electrolyte imbalance before the administration of drugs, particularly correction of hypokalemia to levels of $>4.5 \mathrm{mEq} / \mathrm{L}$ and hypomagnesemia to levels of $>2.2 \mathrm{mg} / \mathrm{dL}$.

- Avoiding other drugs that may cause QT prolongation or have other cardiac adverse effects, if possible (e.g., antiemetics such as ondansetron, proton pump inhibitors for acid suppression, and some anesthetic agents).

- After initiation of a drug that is associated with torsades de pointes, ECG signs indicative of risk for arrhythmia include an increase in QTc from pre-drug baseline of $60 \mathrm{~ms}$, marked QTc interval prolongation $>500 \mathrm{~ms}$, T-U wave distortion that becomes more exaggerated in the beat after a pause, visible (macroscopic) T-wave alternans, new-onset ventricular ectopy, and couplets and nonsustained polymorphic ventricular tachycardia initiated in the beat after a pause.

- Recommended actions when ECG signs of impending torsades de pointes develop are to discontinue the offending drug, administer potassium and magnesium, consider temporary pacing to prevent bradycardia and long pauses, and transfer the patient to a hospital unit with the highest level of ECG monitoring surveillance where immediate defibrillation is available.

- Seriously ill patients on lacosamide should be monitored for significant bradycardia and if so, treated promptly with isoproterenol or atropine and if available external pacing.

In patients with drug-resistant seizures (the second scenario), if there are significant drug-drug interactions and/or adverse effects related to AEDs, the treating physician may decide to switch the AED to a safer drug. In these circumstances, it is important to consider that the altered physiology of critically ill patients as well as pharmacological and nonpharmacological interventions in patients with COVID-19 may lead to therapeutic failure or toxicity of some AEDs [24]. In addition, since these patients are critically ill, a drug with intravenous (IV) formulation is preferable. Furthermore, because these patients suffer from severe respiratory and/or cardiac problems, drugs with significant respiratory/cardiac adverse effects should be avoided. In these settings, levetiracetam and brivaracetam could be safe treatment options. Brivaracetam could be administered 25 to $100 \mathrm{mg}$ twice daily (50-200 mg/day). Dosage adjustment is recommended for all stages of hepatic impairment [25]. Levetiracetam could be administered 30 to $60 \mathrm{mg} / \mathrm{kg}$ IV loading dose over $10 \mathrm{~min}$ and then 1000-3000 mg per day in 2 divided doses. Dosage adjustment is necessary in patients with renal impairment [26].

\section{Organ failure}

Abnormal liver and renal functions may happen in critically ill patients with COVID-19 [27]. In any of these circumstances, AED dosages may need to be adjusted [6].

1. If your patient has moderate (Child-Pugh B) or severe (Child-Pugh C) hepatic impairment, the following AEDs may need dosage adjustment: brivaracetam, cannabidiol, carbamazepine, cenobamate, lacosamide, lamotrigine, perampanel, phenobarbital/primidone, phenytoin, topiramate, and valproate. Even with other AEDs, close 
Table 3 Cardiovascular adverse effects of anti-seizure medications and anti-COVID medications

\begin{tabular}{|c|c|}
\hline & Cardiovascular adverse effects \\
\hline \multicolumn{2}{|l|}{ Anti-seizure medication } \\
\hline Brivaracetam & - \\
\hline Cannabidiol & - \\
\hline Carbamazepine & $\begin{array}{l}\text { Atrioventricular block, cardiac arrhythmias or arrhythmia exacerbation, } \\
\text { and congestive heart failure }\end{array}$ \\
\hline Cenobamate & QT shortening \\
\hline Clobazam & - \\
\hline Clonazepam & - \\
\hline Eslicarbazepine acetate & - \\
\hline Ethosuximide & - \\
\hline Phenobarbital/primidone & May prolong QT interval \\
\hline Phenytoin & Cardiac conduction abnormalities (e.g., bundle-branch block) \\
\hline Valproic acid & - \\
\hline Gabapentin & - \\
\hline Lacosamide & $\begin{array}{l}\text { Prolongation in PR interval, first-degree atrioventricular (AV) block, } \\
\text { second degree, and complete AV blocks }\end{array}$ \\
\hline Lamotrigine & - \\
\hline Levetiracetam & - \\
\hline Oxcarbazepine & - \\
\hline Perampanel & - \\
\hline Pregabalin & Exacerbation of heart failure \\
\hline Rufinamide & QT shortening \\
\hline Topiramate & - \\
\hline Vigabatrin & - \\
\hline Zonisamide & - \\
\hline \multicolumn{2}{|l|}{ Anti-COVID-19 medication } \\
\hline Remdesivir & - \\
\hline Lopinavir/ritonavir & $\begin{array}{l}\text { Bradyarrhythmias, QTc prolongation, AV block, torsade de pointes, and } \\
\text { prolongation of the PR interval }\end{array}$ \\
\hline Chloroquine/hydroxychloroquine & $\begin{array}{l}\text { Direct myocardial toxicity vs. exacerbation of underlying } \\
\text { cardiomyopathy, altered cardiac conduction: QTc prolongation, AV } \\
\text { block, bundle branch block, torsade de pointes, and ventricular } \\
\text { tachycardia/fibrillation }\end{array}$ \\
\hline Interferon beta & $\begin{array}{l}\text { Direct myocardial toxicity vs. exacerbation of underlying } \\
\text { cardiomyopathy, hypotension, arrhythmia, and myocardial infarction }\end{array}$ \\
\hline Favipravir & - \\
\hline Tocilizumab & - \\
\hline Nitazoxanide & - \\
\hline Sofosbuvir & Bradycardia \\
\hline
\end{tabular}

References: [7-16, 18-21]

monitoring may also be warranted to ensure adequate efficacy and limit toxicity [6].

2. If your patient has renal failure with creatinine clearance $<60 \mathrm{~mL} / \mathrm{min}$, the following AEDs may need dosage adjustment: eslicarbazepine acetate, gabapentin, levetiracetam, perampanel, pregabalin, primidone, topiramate, and zonisamide. Even with other AEDs, close monitoring may also be warranted to ensure adequate efficacy and limit toxicity [6].

\section{Extracorporeal membrane oxygenation}

Extracorporeal membrane oxygenation (ECMO), which may become necessary in the management of patients with COVID-19 and severe pneumonia, may potentially affect the pharmacokinetics of highly protein-bound drugs (e.g., clobazam, perampanel, phenytoin, valproate). ECMO circuits may initially sequester these drugs, while later releasing the drug into the circulation resulting in an unpredictable effect 
$[24,28]$. Clinicians should monitor serum drug levels to guide dosing in patients receiving the above AEDs.

\section{New onset seizures or seizure exacerbations}

New onset seizures may happen or seizures may exacerbate in PWE, as a result of acute systemic illness, primary neurological pathology, or medication adverse-effects in critically ill patients with COVID-19 [2, 29, 30]. For example, hydroxychloroquine/chloroquine may lower the seizure threshold [31]. Therefore, the treating physician should be aware of these possibilities and be ready for emergency therapeutic interventions if they happen [2]. For a comprehensive review on this issue, please refer to reference [32].

\section{Conclusion}

The treatment of COVID-19 is a changing landscape. Recently, the Food and Drug Administration (FDA) issued an Emergency Use Authorization (EUA) for emergency use of remdesivir for the treatment of hospitalized COVID-19 patients [33]. Also recently, WHO halted hydroxychloroquine trial over safety concerns $[34,35]$. This manuscript helps healthcare professionals understand the potentials for drug interactions between AEDs and therapies suggested and used in COVID-19. Drug-drug interactions and adverse drug reactions of AEDs and anti-COVID-19 therapies could pose significant therapeutic challenges. While not all potential drugdrug interactions are clinically significant, some may represent a tremendous threat to the well-being of patients and should be considered seriously in the management process of critically ill patients. In addition, cardiac, hepatic, or renal impairments in patients with COVID-19 may require adjustment to AEDs. Therapeutic drug monitoring and also close monitoring of the patient's clinical status is necessary when PWE contract COVID-19 and need to receive treatment. We should emphasize that covering all drugs used in the treatment of patients with epilepsy was beyond the scope of this review. For example, while steroids and everolimus are not conventional AEDs, they may be used in the treatment of some epilepsy syndromes, such as autoimmune epilepsies and tuberous sclerosis complex. Corticosteroids may mask some signs of infections and may reduce resistance to new infections [36]. Furthermore, corticosteroids may exacerbate infections and increase the risk of disseminated infections [36]. Similarly, everolimus has immunosuppressive properties and may predispose patients to bacterial, fungal, or viral infections. Furthermore, everolimus may cause non-infectious pneumonitis (presenting with non-specific respiratory signs and symptoms such as hypoxia, cough, and dyspnea) [37]. Therefore, it is important to consider any and all adverse effects and drug interactions in patients with epilepsy, who become infected with SARS-CoV2 and need treatment for COVID-19.

Authors' contributions Ali A. Asadi-Pooya, MD: study design, review process, and manuscript preparation. Armin Attar, MD, PhD; Mohsen Moghadami, MD; Iman Karimzadeh, PharmD, PhD: review process and manuscript preparation.

\section{Compliance with ethical standards}

Conflict of interest Ali A. Asadi-Pooya received honoraria from Cobel Daruo, RaymandRad, and Tekaje; and a royalty from Oxford University Press (book publication). The other authors declare that they have no conflict of interest.

Ethical approval None

\section{References}

1. Rothan HA, Byrareddy SN (2020) The epidemiology and pathogenesis of coronavirus disease (COVID-19) outbreak. J Autoimmun 109:102433

2. Asadi-Pooya AA, Simani L (2020) Central nervous system manifestations of COVID-19: a systematic review. J Neurol Sci 413: 116832

3. https://www.ilae.org/patient-care/covid-19-and-epilepsy/covid-19information-for-clinicians/faqs-for-clinicians/ Accessed on April 18,2020

4. Patsalos PN (2013) Drug interactions with the newer antiepileptic drugs (AEDs) - part 1: pharmacokinetic and pharmacodynamic interactions between AEDs. Clin Pharmacokinet 52:927-966

5. Perucca E (2005) Clinically relevant drug interactions with antiepileptic drugs. Br J Clin Pharmacol 61:246-255

6. Asadi-Pooya AA, Sperling MR (2016) Antiepileptic drugs: a clinician's manual, Second edn. Oxford University Press, New York

7. https://www.drugbank.ca/drugs/DB06218/ Accessed on April 18, 2020

8. http://products.sanofi.ca/en/plaquenil.pdf/ Accessed on April 21, 2020

9. https://www.accessdata.fda.gov/drugsatfda_docs/label/2016/ 125276s107_125472s0181bl.pdf/ Accessed on April 21, 2020

10. Nguyen T, McNicholl I, Custodio JM, Szwarcberg J, Piontkowsky D (2016) Drug interactions with cobicistat- or ritonavir-boosted Elvitegravir. AIDS Rev 18:101-111

11. https://www.webmd.com/interaction-checker/default.htm/ Accessed on April 19, 2020

12. https://www.accessdata.fda.gov/drugsatfda_docs/label/2007/ 021226s018lbl.pdf/ Accessed on April 19, 2020

13. https:/www.accessdata.fda.gov/drugsatfda_docs/label/2015/ 204671s004lbl.pdf/ Accessed on April 21, 2020

14. Huang CW, Brown S, Pillay N et al (2018) Electroencephalographic and electrocardiographic effect of intravenous lacosamide in refractory focal epilepsy. J Clin Neurophysiol 35:365-369

15. https://www.covid19-druginteractions.org/ Accessed on April 21, 2020

16. https://www.accessdata.fda.gov/drugsatfda_docs/label/2005/ 021818lbl.pdf/ Accessed on April 21, 2020

17. van der Lee MJ, Dawood L, ter Hofstede HJ, de Graaff-Teulen MJ, van Ewijk-Beneken Kolmer E, Caliskan-Yassen N, Koopmans PP, Burger DM (2006) Lopinavir/ritonavir reduces lamotrigine plasma concentrations in healthy subjects. Clin Pharmacol Ther 80:159 168 
18. Dhatt MS, Gomes JA, Reddy CP, Akhtar M, Caracta AR, Lau SH, Damato AN (1979) Effects of phenytoin on refractoriness and conduction in the human heart. J Cardiovasc Pharmacol 1:3-18

19. Kenneback G, Bergfeldt L, Vallin H, Tomson T, Edhag O (1991) Electrophysiologic effects and clinical hazards of carbamazepine treatment for neurologic disorders in patients with abnormalities of the cardiac conduction system. Am Heart J 121:1421-1429

20. https://reference.medscape.com/drug/xcopri-cenobamate-1000328/ Accessed on April 18, 2020

21. Roden DM, Harrington RA, Poppas A, Russo AM (2020) Considerations for drug interactions on QTc in exploratory COVID-19 (Coronavirus Disease 2019) treatment. J Am Coll Cardiol. https://doi.org/10.1016/j.jacc.2020.04.016

22. Chen T, Wu D, Chen H, Yan W, Yang D, Chen G, Ma K, Xu D, Yu $\mathrm{H}$, Wang H, Wang T, Guo W, Chen J, Ding C, Zhang X, Huang J, Han M, Li S, Luo X, Zhao J, Ning Q (2020 Mar 26) Clinical characteristics of 113 deceased patients with coronavirus disease 2019: retrospective study. BMJ. 368:m1091. https://doi.org/10. 1136/bmj.m1091

23. Drew BJ, Ackerman MJ, Funk M, Gibler WB, Kligfield P, Menon V, Philippides GJ, Roden DM, Zareba W, American Heart Association Acute Cardiac Care Committee of the Council on Clinical Cardiology, Council on Cardiovascular Nursing, American College of Cardiology Foundation (2010) Prevention of torsade de pointes in hospital settings: a scientific statement from the American Heart Association and the American College of Cardiology Foundation. J Am Coll Cardiol 55:934-947

24. Farrokh S, Tahsili-Fahadan P, Ritzl EK, Lewin JJ 3rd, Mirski MA (2018) Antiepileptic drugs in critically ill patients. Crit Care 22:153

25. Brigo F, Lattanzi S, Nardone R, Trinka E (2019) Intravenous brivaracetam in the treatment of status epilepticus: a systematic review. CNS Drugs 33:771-781

26. Trinka E, Kälviäinen R (2017) 25 years of advances in the definition, classification and treatment of status epilepticus. Seizure 44 : $65-73$
27. Zhu J, Ji P, Pang J, Zhong Z, Li H, He C, Zhang J, Zhao C (2020 Apr 15) Clinical characteristics of 3,062 COVID-19 patients: a meta-analysis. J Med Virol. https://doi.org/10.1002/jmv.25884

28. Wang D, Hu B, Hu C, Zhu F, Liu X, Zhang J, Wang B, Xiang H, Cheng Z, Xiong Y, Zhao Y, Li Y, Wang X, Peng Z (2020 Feb 7) Clinical characteristics of 138 hospitalized patients with 2019 novel coronavirus-infected pneumonia in Wuhan, China. JAMA 323: 1061. https://doi.org/10.1001/jama.2020.1585

29. Niazkar HR, Zibaee B, Nasimi A, Bahri N (2020) The neurological manifestations of COVID-19: a review article. Neurol Sci. https:// doi.org/10.1007/s10072-020-04486-3

30. Iroegbu JD, Ifenatuoha CW, Ijomone OM (2020) Potential neurological impact of coronaviruses: implications for the novel SARSCoV-2. Neurol Sci 41:1329-1337

31. https://www.accessdata.fda.gov/drugsatfda_docs/label/2017/ 009768s 037s045s 047lbl.pdf/ Accessed on April 21, 2020

32. Asadi-Pooya AA (2020) Seizures associated with coronavirus infections. Seizure 79:49-52

33. https://www.fda.gov/media/137564/download/ Accessed on May 30, 2020

34. https://www.npr.org/sections/coronavirus-live-updates/2020/05/ 25/861913688/who-halts-hydroxychloroquine-trial-over-safetyconcerns/ Accessed on May 30, 2020

35. Mehra MR, Desai SS, Ruschitzka F, Patel AN (2020) Hydroxychloroquine or chloroquine with or without a macrolide for treatment of COVID-19: a multinational registry analysis. Lancet S0140-6736(20):31180-31186

36. https://www.accessdata.fda.gov/drugsatfda_docs/label/2012/ 202020s000lbl.pdf/ Accessed on June 19, 2020

37. https://www.accessdata.fda.gov/drugsatfda_docs/label/2012/ 022334s016lbl.pdf/ Accessed on June 19, 2020

Publisher's note Springer Nature remains neutral with regard to jurisdictional claims in published maps and institutional affiliations. 